\title{
Facial recognition techniques using SVM: A comparative analysis
}

\section{(Técnicas de reconocimiento facial usando SVM: un análisis comparativo)}

\author{
José Augusto Cadena Moreano ${ }^{1}$, Nora La Serna Palomino², Alex Christian Llano Casa ${ }^{3}$
}

\begin{abstract}
This paper presents a literary review of facial recognition in 2D, which plays an important role in the life of the human being in terms of safety, work activity, etc. The focus is on the results obtained by some researchers with the application of feature extraction techniques, pattern classifiers, databases and their respective percentage of efficiency obtained. The objective is to determine efficient techniques that allow an optimal $2 D$ facial recognition process, based on the quality of databases, feature extractors and pattern classifiers.
\end{abstract}

\section{Keywords}

Databases, Support vector machine, Facial recognition, Artificial neural networks.

\section{Resumen}

Este trabajo presenta una revisión literaria sobre reconocimiento facial en 2D, la cual juega un papel importante en la vida del ser humano en cuanto a su seguridad, actividad laboral, etc. El enfoque está en los resultados obtenidos por algunos investigadores con la aplicación de técnicas de extracción de características, clasificadores de patrones, bases de datos y su respectivo porcentaje de eficiencia obtenida. El objetivo es determinar técnicas eficientes que permitan realizar un proceso óptimo de reconocimiento facial en 2D, en función de la calidad de bases de datos, extractores de características y clasificador de patrones.

\section{Palabras clave}

Bases de datos; máquina de vectores soporte; reconocimiento facial, redes neuronales artificiales.

\section{Introduction}

In recent years, the security of many countries has been compromised by terrorist attacks, kidnapping, and thefts, especially in nations with great political and economic power, as is the case of the United States of America. The terrorist attack on September 11 (2001), widely known around the world, demonstrated the weaknesses in the intelligence and national security systems, and since then the Government has invested in improving these security systems at airports, intelligence agencies, public buildings, banks, educational institutions, etc. One of the areas that has been highly promoted is facial recognition, especially in recent years.

Facial recognition systems currently have high recognition rates, but still presents some errors, due to the multiple variables and complexity of the human face.

Humans have the ability to recognize themselves and others without any difficulty, before they engage in any kind of activity, using facial traits; even more, individuals can recognize the face of any person after a long time not seeing him/her. Different ages, races, far or near, with poor or good lighting, regardless the circumstance, there is always the ability to recognize a face based 
on previous experiences or memories. However, it is necessary to automate this activity, since it is impossible for to be at the time and place when some action occurred, such as thefts, murder, kidnapping, etc. Hence the need for a computer-controlled facial recognition system.

However, the facial recognition of human faces has still some difficulties that challenges computer's systems and hasn't yet reach the expected efficiency.

As part of the computer vision in which the 3D Vision is inserted (Moreno, 2004), researchers today work on systems that avoid restrictions on controlled conditions to acquire robustness in light, age, rotation, translation, depth, facial expressions, occlusions, hair, mask, make-up, etc.

To solve the problems that facial recognition in $2 \mathrm{D}$ arises there are some techniques, according to González (2007), that work through a 3D perspective. The advantage of using 3D data (representing $180^{\circ}$ images in cylindrical coordinates) is that in addition to the texture, we have depth information and the recognition system is more robust against changes in lighting, pose and expression because the depth does not change, even if these aspects of the image do. On the other hand, according to Del Alamo, C., Romero, L. \& Fuentes, L. (2015), analyzing or comparing 3D models with non-rigid transformations could be costly, especially if the models are represented by triangular meshes with a large number of vertices and edges.

A facial recognition system typically follows the subsequent steps, as shown in Figure 1:

- Acquisition of images: have a database of human faces.

- Screening of the face in the image: identify the presence of a face in a photo image.

- Feature extraction: extracts all information from characteristic features of the person's face and;

- Recognition: process of identifying the person.

Figure 1. Primary stages of a facial recognition system

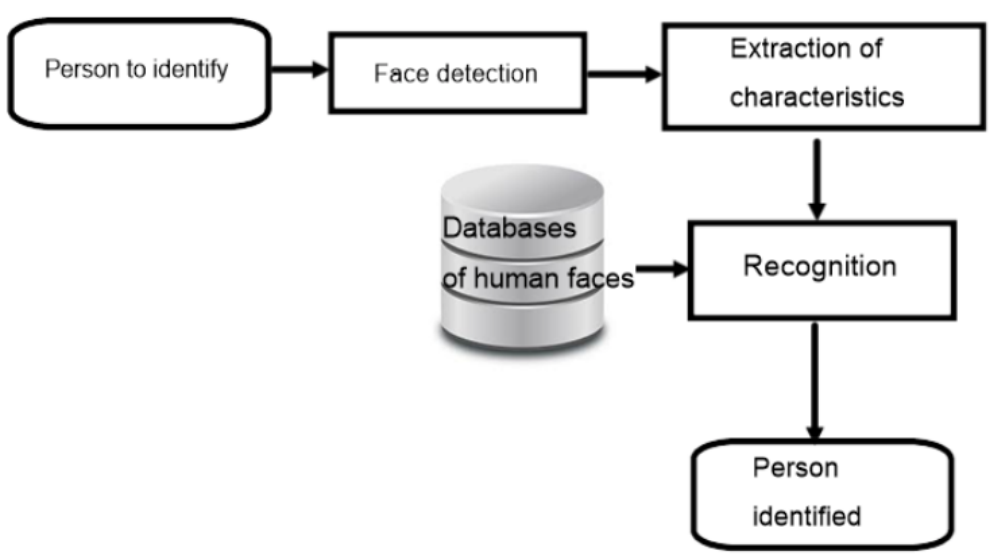

So there are now several techniques that allow the treatment of recognition.

This work aims at a comprehensive analysis of the work on facial recognition based on the use of some main techniques, such as: PCA (Main Components Analysis) (Moreno \& Sanchez, 2005), which allows to reduce the dimensionality of an image, and the SVM (Support Machines Vectors), considered as pattern classifier. These SVMs (Correa, Salazar, \& Ortiz, 2013) appeared in the 1990s as an optimal classification method, consisting of a set of supervised learning algorithms developed by Cortes \& Vapnik (Vapnik, 1998). 
Cabello (2003) reviews classifiers, considering it as algorithms that allow to define a model for each class, so that the class to which an element belongs can be calculated from the values of the data that define the element.

The aim is also to review certain databases used and to determine the most effective technique based on the results obtained and, at the same time, identify the main research trends in this area.

\section{Related Work}

\section{Facial recognition using 3D geometric characteristics: PCA and SVM}

This work presents a 3D face modeling system based on a HK segmentation algorithm, which consists on separating the face in regions with established characteristics (Moreno \& Sanchez, 2005). Six regions and two lines were automatically obtained from each 3D mesh. These are used to obtain the most relevant facial trait, according to the coefficient of Fisher (Correa, Salazar, \& Ortiz, 2013), which allows to find regions of the face. The robustness of this model has been proven by the implementation of two PCA-based facial recognition systems (Moreno \& Sanchez, 2005), a technique to reduce the dimensionality of a set of images and Support Machines Vectors (SVM) as pattern classifiers, taking into account the form as a matching scheme under control and uncontrolled environments. Experimental results show that the correct recognition rates are sufficient to implement real-face recognition applications and that the proposed model is robust. The use of SVMs in an uncontrolled environment produces $77.9 \%$ of the recognition rate, while $90.16 \%$ is obtained in a controlled environment. PCA in an uncontrolled environment is $76.2 \%$ and $82 \%$ in a controlled environment. The GAVAB database (Moreno y Sánchez, 2004), containing 420 3D facial images of 60 different individuals, is used in this research.

\section{Support vector machines for recognition}

This work deals with multi-class facial recognition (Guo, Li, \& Chan, 2001), and specifies that a learning network method can be used for recognition of bipartite patterns; a binary tree recognition strategy is incorporated into SVM to handle the multi-class facial recognition problem. On the other hand, the performance of the SVM is based on comparison with the standard eigenface approach, and its latest algorithm proposes the closest characteristic line (NFL) (Guo et al., 2001), (Peng, 2010), Pradhan, 2013), (Z. Qi, Y. Tian, 2013), which is an efficient but simple classification method for pattern recognition.

This work presents a strategic binary tree for the SVM to solve the multi-class recognition problem as shown in Figure 2.

If you plan to build a binary tree for classification, there you have eight classes. The numbers from 1 to 8 are the ones that encode the classes; it is worth pointing out these numbers because coding can be arbitrary. Then you choose a winning number that comes out of the comparison between each pair of classes. The winning or selected classes will reach the top level for another test round. Guo uses three classification methods: Line nearest features (NFL) (NFL), Nearest Centre (NC) (closest centre) and SVM (Guo et al., 2001). Two experiments yielded the errors in recognition, the first working with an ORL database, with a total of 40 different participants with 10 poses each, obtaining a $3 \%$ error for the SVM, which was the minimum compared to 3,83 \% for CNN, and 3,125\% NFL; and a second experiment with 1079 images of 
137 persons corresponding to a database composed of 5 databases, such as: ORL, BERN, YALE among others, of which it is concluded that the SVM has the lowest error in recognition (8,79\%), followed by NFL (9,72 \%) and NC (15,14\%).

Figure 2. Structure of a binary tree for 8 classes of facial recognition. (Guo et al., 2001)

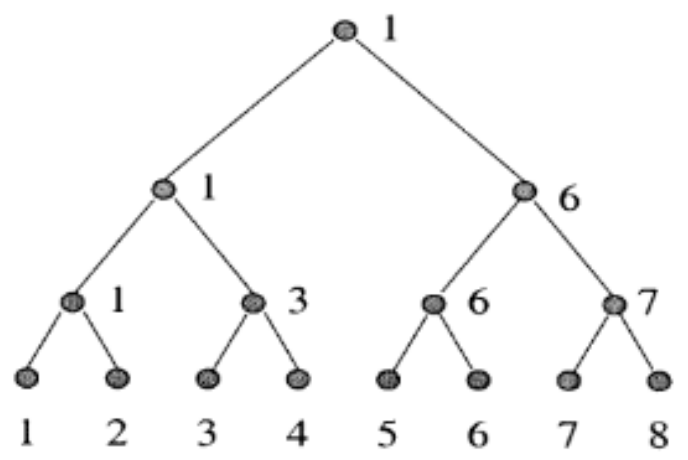

Fig. 2. The binary tree structure for 8 classes face recognition. For a coming test face, it is compared with two pairs each, and the winner will be tested in an upper level until the top of the tree. The numbers 1-8 encode the classes.

\section{An improved discriminative common vectors and SVM based face recognition approach}

An improved method of discriminatory common vectors, Improved Discriminative Common Vectors (IDCV) used by SVM, is proposed (Wen, 2012), and to demonstrate their efficiency they compare seven other facial recognition methods, two of which include SVM. For experiments they use the "The ORL Database of Faces" and "The AR-face database" face imaging databases, as shown in Figures 3 and 4, respectively.

Figure 3. ORL Databases

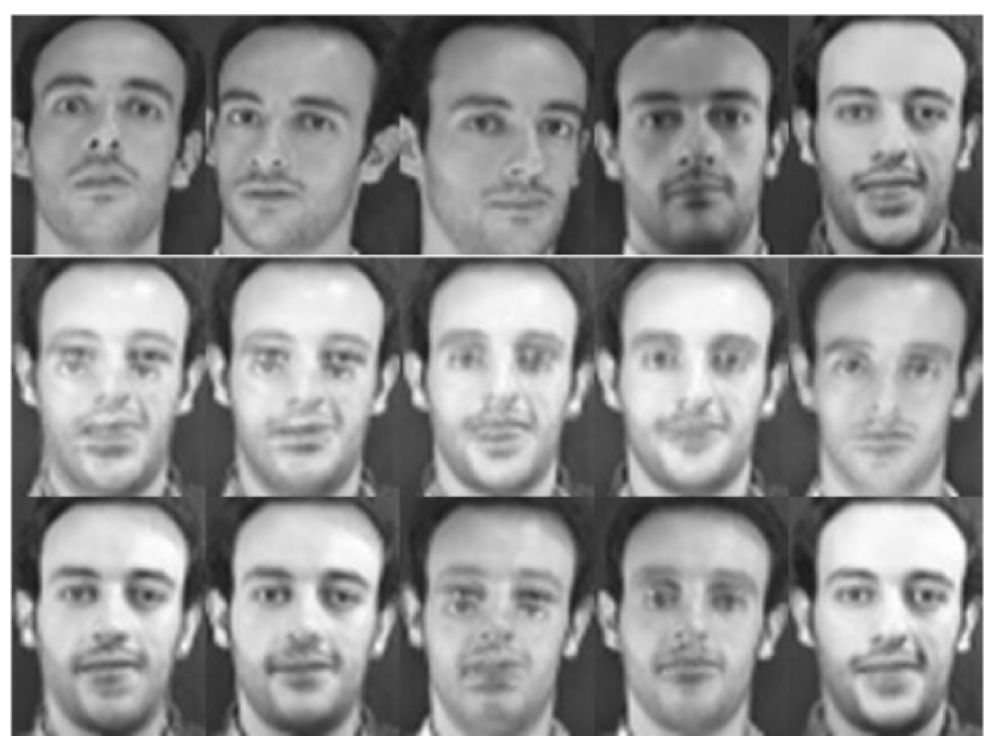


Figure 4. AR databases
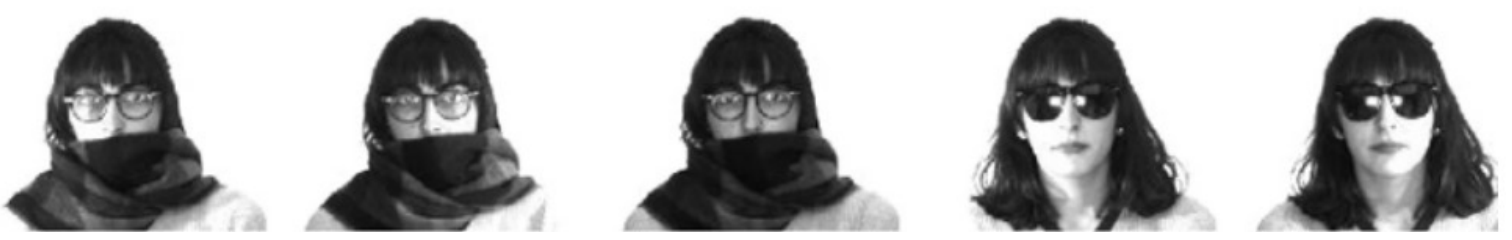

When working with ORL, uses 400 images of 40 people. Each image is 112 by 92 and the person on it has 10 different poses. While in working with AR uses 1300 images, 50 female and 50 male, 13 poses per person. This work shows that the proposed IDCV technique with SVM has a higher recognition rate than the other seven methods, with each of the two databases used. Common vectors are generated in the event that the number of individuals in each class is less than or equal to the dimensionality of the space of individuals.

The Common Discriminatory Vectors (CSD) algorithm is a variation of the Linear Discriminatory Analysis (LDA) algorithm, which shows that CSD achieves better facial recognition results than LDA. However, it does not solve the multiclass problem of the LDA algorithm. The author proposes the Better Common Discriminant Vectors (IDCV) method, adjusting the Fisher criterion of LDA in CSDs, to estimate the dispersion matrices between classes and within the class.

This study uses SVM to classify training and test data, with the Gaussian kernel function. Table 1 shows the results obtained in the experiments carried out for seven trainings, where the IDCV technique with SVM presents the highest performance, as well as a summary of the best results obtained under these techniques studied.

Table 1. Summary of results obtained for seven trainings with 8 techniques (Wen, 2012)

\begin{tabular}{|c|c|c|}
\hline TECHNIQUES & EFFICIENCY & KERNEL \\
\hline EIGENFACE & $93,87 \%$ & \multirow{8}{*}{ Gaussian } \\
\hline LDA & $93,8 \%$ & \\
\hline PCA-SVM & $95,56 \%$ & \\
\hline DCV-NN & $96,67 \%$ & \\
\hline KDCV-NN & $97,75 \%$ & \\
\hline DCV-RBF & $97,77 \%$ & \\
\hline KDCV-RBF & $97,65 \%$ & \\
\hline IDCV-SVM & $98,92 \%$ & \\
\hline
\end{tabular}

\section{A New Face Recognition Method Based on Fast Least Squares Support Vector Machine}

This work (Kong \& Zhang, 2011) proposes a new method for facial recognition by combining Independent Component Analysis (ICA) (Bartlett, 1999) and SVM. To do this, initially extracts features from the faces using the Informax algorithm, then it implements face recognition using the tiny square quick algorithm (FLS-SVM). ICA is a statistical signal processing technology (1990) that can extract image features from faces. It is a technique for extracting statistically indepen- 
dent signals from a mixture of signals. The idea of ICA is to represent a set of random variables using basic functions, where the components are statistically as independent as possible.

The experiment validates the facial recognition algorithm using the ORL database. This BD includes images of 40-person faces 28-by-28, all have ten facial images (different expression, with glasses or not glasses). Finally, five images are selected at random as a training set, and others as a test set. All images are cropped to size 28 by 28 and rectified according to the positions of the eyes manually placed. The results obtained in your experiment are presented in Table 2.

Table 2. Summary of results obtained for seven trainings with 8 techniques (Wen, 2012)

\begin{tabular}{|c|c|c|c|c|}
\hline \multirow{2}{*}{$\begin{array}{c}\text { Method of } \\
\text { extracting } \\
\text { features }\end{array}$} & \multicolumn{2}{|c|}{ classifier } & $\begin{array}{c}\text { The } \\
\text { number of } \\
\text { principal } \\
\text { component }\end{array}$ & $\begin{array}{c}\text { Correct } \\
\text { recognition } \\
\text { ratio(\%) }\end{array}$ \\
\hline \multirow{2}{*}{ PCA } & \multirow{2}{*}{ FLS-SVM } & $\begin{array}{c}\text { PK } \\
(\mathrm{d}=2)\end{array}$ & 80 & 94.50 \\
\cline { 3 - 5 } & \multirow{2}{*}{ FL } & $\begin{array}{c}\mathrm{PK} \\
(\mathrm{d}=3)\end{array}$ & 80 & 95.50 \\
\cline { 3 - 5 } & \multirow{2}{*}{$\begin{array}{c}\mathrm{GK} \\
(\mathrm{d}=0.7)\end{array}$} & 80 & 96.00 \\
\hline \multirow{2}{*}{ ICA } & $\begin{array}{c}\mathrm{PK} \\
(\mathrm{d}=2)\end{array}$ & 60 & 96.00 \\
\cline { 3 - 5 } & & $\begin{array}{c}\mathrm{PK} \\
(\mathrm{d}=3)\end{array}$ & 60 & 96.50 \\
\cline { 3 - 5 } & & $\begin{array}{c}\mathrm{GK} \\
(\mathrm{d}=0.7)\end{array}$ & 60 & 97.50 \\
\hline
\end{tabular}

Based on the results obtained, it is shown that ICA with the FLS-SVM classifier was the most effective, with a maximum recognition of $97.5 \%$.

\section{Face Recognition using R-KDA with Non-Linear SVM for Multi-View Database}

This work (Devi, Laishram, \& Thounaojam, 2015), aims to develop a reconnaissance application by combining R-KDA with non-linear SVMs, and in turn the results are compared with the results of neighbouring R-KDA and K-nn respectively, achieving the best results for the former. The work proposes a two-phase recognition system: The feature extraction phase and the face recognition phase, for the former, uses R-KDA and non-linear SVM for the second.

Similarly, a series of steps for recognition, the same as using the SVM classifier, finds an optimal hyperplane that the largest possible fraction of the points in the same class; on the same side, it maximizes the distance from any class to the hyperplane. Determining the optimal hyperplane is a limited optimization problem and can be solved using quadratic programming techniques.

The UMIST database used consists of 564 images of 20 people. Each covers a series of front profile poses of views. Subjects cover a range of race, sex, appearance. The files are in pgm format, approximately $220 \times 220$ pixels of 256 shades of gray. For the experimental purpose, 18 subjects were taken in 10 different poses.

Finally, based on the result of the analysis, it is observed that the combination of R-KDA with non-linear SVM exceeds the combination of R-KDA with k-nn. The best correct recognition 
rate achieved with this proposed algorithm is approximately $30 \%$ and the kernel function used is the radio base function (RBF), taking into account that it is a set of multi-view faces.

\section{Modifications to the Eigenphases Method for Face Recognition Based on SVM}

The authors (Olivares-Mercado, Toscano-Medina, Sánchez-Pérez, Nakano-Miyatake, \& PérezMeana, 2016) submit two modifications to the eigenphases method, in order to increase their accuracy. The first modification is called Local Space Domain Eigenfaces (LSDE), where the face image is first segmented into blocks of $\mathrm{N} \times \mathrm{N}$ pixels, whose magnitudes normalize. The second modification is known as local frequency domain eigenphase (LFDE), where after segmentation the pixel magnitudes are normalized and the phase spectrum is calculated independently; then all the blocks are concatenated and PCA is applied (Benitez-Garcia, OlivaresMercado, Aguilar-Torres, Sanchez-Perez, \& Perez-Meana, 2012) to reduce the dimensionality of the problem.

The AR database (Robert, 2014), with 9360 faces, is used and works with 120 persons (65 men and 55 women). It performs two different trainings with 1200 images each. Also, to implement the SVM uses the LIBSVM library (Chang \& Lin, 2013), and the function used for the training and reconnaissance task is the Polyneal Kernel. As for identification using SVM, it uses algorithms such as: Gabor, Wavelet, LSDE 3, LSDE 6, LFDE 3, LFDE 6, distributed in two groups or sets. It is noted that LFDE 3 is the most successful: the first set has a success rate of $85.92 \%$ and the second set is $97.92 \%$ correct.

\section{Facial expression recognition based on the fusion feature of PCA and LBP with SVM}

Work on Primary Component Analysis (PCA), which is a method of extraction based on statistical characteristics of global grayings of the entire image. The authors (Luo, Wu, \& Zhang, 2013), introduce a hybrid method of major component analysis and a local binary pattern (LBP). LBP extracts local grayscale characteristics from the mouth region, which contribute more to the recognition of facial expressions, to help the global characteristics of the grayscale in the recognition of facial expressions.

The recognition is designed based on the VC6.0 environment, and the input device is a PHLIPS camera. In this experiment, the set of training samples include seven different facial expressions, and the sum of them is 50 . Half of them are better lit and the others are less illuminated, and each image of the training set was standardized to a small size of $24 \times 24$.

A comparison is made between PCA+SVM and PCA+LBP+SVM. Among them, an average recognition was obtained of: for PCA+SVM the highest was $93.75 \%$ for neutral expression and for combination PCA+LBP+SVM $96.25 \%$ recognition for both neutral and cheerful expression, both using an RBF kernel.

\section{Face recognition using support vector model classifier for user authentication}

This work (Lin, Wang, \& Tsai, 2016) developed an online facial recognition system by forming an SVM classifier based on the user's facial features associated with the transform and a spatially improved local binary pattern. This cross-validation system and associated SVM used the Olivetti Research Laboratory database of facial features to troubleshoot classification accuracy issues. 
Experimental results showed that the classification error decreased with an increase in the size of training samples. Hence, the overall accuracy of face recognition is more than $97 \%$, for a data size of 168 and 341 in low and high resolution images, respectively. Regarding the transformation, it is stated that it should allow changes only in the length of time, but not in the form, based on appropriate base functions. The most common facial features appear as part of low resolution information, while the high resolution part contains most facial features, such as local variations in lighting, expression and clothing. Conversely, the reverse wavelet transformation combines the two parts of the face image in the original image through a process of rebuilding information.

This research uses 3 kernels functions: Linear, Polynomial and RBF (Radial base function), where the Linear, both in training and testing, has being the most successful. Compared to existing systems, the system has a number of important advantages, such as:

a) Provides the benefit of comprehensive privacy protection through distributed storage, where smart card stores images of different facial expressions and a database contains image records of the face.

(b) The model uses several layer analytical diagrams for wave conversion and a dual pattern of a spectrum of a spatially improved histogram with a LBP function, and improves the accuracy of facial recognition.

\section{SVM-based feature extraction for facial recognition}

In this work (Kim, Park, Toh, \& Lee, 2010) the goal is to find an effective sub-space for identity discrimination that will serve to extract features from the face. This has been extended for non-linear hypersurface; however, there are still limitations for non-linear ADL to address physical applications under complex environmental factors. These limitations include the use of a common covariance function between each class, and the limited dimension inherent in defining the dispersion between the class, since these problems are inherently caused by Fisher's own definition of criteria, which cannot be solved in the conventional LDA framework (Kim et al., 2010). It uses a non-linear kernel, a polynomial, in this case.

Considering this situation, it proposes to adopt a dispersion based on the margin between the class and a regularization process to solve the problem. For this purpose, it redesigns the dispersion matrix between the class based on the SVM margins to facilitate the extraction of characteristics in an effective and reliable manner. Following this is a regularization of the dispersion matrix within the class. In this process some empirical experiments are carried out to compare the proposed method with several other variants of the LDA method using the FERET (P.J. Phillips, H. Moon, S. Rizvi, 2000), AR (Robert, 2014) and CMU-PIE (Sim, Baker, \& Bsat, 2003) databases 113. Three experiments are carried out with these databases respectively. With FERET (1702 images) corresponding to 256 people, with AR (1680 images) corresponding to 120 people and finally with CMU-PIE (1840 images) corresponding to 68 people. All images were 56 x 46 pixels in size. SVM-DA (SVM-based Discriminatory Analysis) error rate is the minimum in relation to KFD (discriminating Kernel Fisher), GDA (Generalized Discriminatory Analysis), KDDA (Direct Discriminatory Analysis) techniques. The best results obtained was the one with the three databases (FERET, ARE, CMU.PIES); the SVM-DA method achieved the lowest errors such as: $8.5 \%, 8.6 \%$ and $9.5 \%$, respectively. 


\section{Evaluation of face recognition using PCA, wavelets and SVM}

Here is an assessment of the use of different face recognition methods (Gumus, Kilic, Sertbas, $\&$ Ucan, 2010). It uses features extraction techniques such as wavelet decomposition and the Eigenfaces method that is based on Primary Component Analysis (PCA). After generating characteristic vectors and distance classifiers, it uses supporting vector machines (SVMS) for the classification stage. Examines the accuracy of the classification according to the increase in the size of the training set and uses a function of the chosen core for the SVM classifier. As a test team, it uses the ORL Face Database (ORL, 1994), known as a standard face database for facial recognition applications that include 400 images of 40 people. At the end of the overall separation task, the accuracy of the Wavelet-SVM approach classification of $98.1 \%$ was obtained for the training of 240 images, taking into account Wavelet-based weighted means of recognition that yielded better results than the PCA-based approach.

Recognition rates were also compared according to gender and noted that male individuals have a higher recognition rate (89.38 \%) than female (81.41\%).

\section{Multi-objective uniform design as a SVM model selection tool for face recognition}

According to the authors (Li, Liu, \& Gong, 2011), the main difficulty in selecting the medium vector machine (SVM) model is a high computational cost. The author first proposes a multipurpose uniform design (MOUD) search method as a SVM model selection tool, and then applies this SVM classifier optimized for face recognition. By replacing the single objective criterion with multi-objective criteria and adopting a uniform design to search for experimental points that are evenly dispersed throughout the experimental domain, the MOUD can reduce computational cost and improve classification capacity simultaneously.

Experiments are run on the UCI benchmark using the Yale face databases (Deng, 2008) and CAS-PEAL-RI (Gao, W., Cao, B., Shan, S., Chen, X., Zhou, D., Zhan, 2008). Experimental results show that the proposed method exceeds significantly other model search methods, especially for facial recognition. In addition to the databases used, the functionality of the polynomial core was also used.

According to the results, with the two databases used (YALE with 165 images and CASPEAL-RI with 100 images), MOUD, as a SVM model selection tool, can effectively alleviate the computational cost of selecting the SVM model, and, at the same time, can increase the capacity of facial recognition; in this case, the major rating result of $96.67 \%$ was achieved.

\section{Kernel subclass convex hull sample selection method for SVM on face recognition}

According to the authors (Zhou, Jiang, Tian, \& Shi, 2010), the SVM has a vital flaw: it needs a large calculation for large-scale learning tasks, for which they propose a selection of samples to overcome this problem. In order to reduce training samples without sacrificing recognition accuracy, this work introduces a new sample selection method called Kernel Subclass Convex Hull (KSCH), which attempts to select limit samples for each convex class hull. This sample selection method is more suitable for non-linear SVM, which works with three kernels: Linear, Quad and RBF.

Therefore, samples selected by this method can efficiently represent the original training set and support the classification of SVMs. For the final experimental results MIT-CBCL facial 
databases (Weyrauch, Heisele, \& Blanz, 2004) and UMIST (Graham \& Allinson, 1998) were used to verify that the KSCH sample selection method can select fewer high quality samples to maintain the recognition accuracy of SVM. Finally, with this $\mathrm{KSCH}$ method you can reduce the size of the training set at the shortest run time with a high classification precision. In this sense, training is carried out with 180 images for the three kernels out of a total of 1032 images, with a classification requirement of $100 \%$.

\section{A novel SVM+NDA model for classification with an application to face recognition}

In this research paper (Khan, Ksantini, Ahmad, \& Boufama, 2012) the authors agree on the fact that SVM is a powerful classification methodology. This work introduces a new SVM + NDA (NonParametric Discriminatory Analysis) model which can be seen as an extension to the SVM by incorporating some partially comprehensive information, especially discriminatory information in the normal direction of the border decision. This can also be seen as an extension of the NDA where support vectors improve the choice of nearest k-neighbors at the decision limit by incorporating local information.

As an extension to both SVM and NDA, you can treat heteroscedastic and non-normal data, as well as avoid the small problem of sample size. It is proposed to use a kernel model called KSVM + KNDA to deal with non-linear problems with real databases.

In general, it can be observed that the SVM+NDA technique has the best results in terms of precision, with $98 \%$ of the data being reviewed in the databases. Thus, work was done on the ORL database (Yu, Yang, $\&$ a, 2001) with a total of 400 front images of 40 people, ranging in pose, lighting, expression and facial details. Also uses the YALE database (Georghiades, 1997), with 165 images, as well as the ESD database SEX (Spacek, 2008) with 153 people (20 images for each) and finally the JAFFE database (Lyons, 1999) with 213 images.

\section{Face Recognition Using Neural Network: A Review}

This work (Kasar, Bhattacharyya, \& Kim, 2016) considers a neural network to be a very powerful and robust classification technique that can be used to predict not only known data, but also unknown data. A neural network is used in many areas, such as visual stage interpretation, speech recognition, face recognition, fingerprint recognition, iris recognition, etc. This research work compares some neural network techniques for facial recognition, making it possible for the fusion between the backward propagation network and the radial base function (BNP and $\mathrm{RBF}$ ) to reach $97.56 \%$ effectiveness in facial recognition.

Neural networks are classification and prediction techniques widely used to facial recognition in other areas such as: education (Calle-López, Cornejo-Reyes, Pesántez-Avilés, \& Rodas, 2018), electricity (Michel, Aleaga, Fernando, Garcés, \& García, 2015), (Benavides, Jurado, \& González, 2018), business (Valdes, Aleaga, \& Vidal, 2014), among others.

After reviewing the main input of several facial recognition-based articles, the results are summarized in Table 3. 
Table 3. Summary of key results obtained

\begin{tabular}{|c|c|c|c|c|c|}
\hline Authors & $\begin{array}{l}\text { Techniques } \\
\text { Used }\end{array}$ & $\begin{array}{l}\% \text { Correct } \\
\text { recognition }\end{array}$ & Databases & $\begin{array}{l}\text { Number of } \\
\text { images }\end{array}$ & Size (pixels) \\
\hline \multirow{2}{*}{$\begin{array}{l}\text { Moreno et al., } \\
(2005)\end{array}$} & PCA & $77.9 \%$ & \multirow{2}{*}{ Gavab } & \multirow{2}{*}{420} & \multirow{2}{*}{---} \\
\hline & SVM & $76.2 \%$ & & & \\
\hline \multirow{6}{*}{ Guo et al,. 2001} & SVM & $\begin{array}{l}3.0 \% \text { (el más mínimo } \\
\text { error) }\end{array}$ & \multirow{3}{*}{ ORL } & \multirow{3}{*}{400} & \multirow{3}{*}{---} \\
\hline & CNN & $3.83 \%$ (de error) & & & \\
\hline & NFL & $3.125 \%$ (de error) & & & \\
\hline & SVM & $8.79 \%$ (de error) & \multirow{3}{*}{$\begin{array}{ll}\mathrm{B} & \mathrm{D} \\
\text { COMPUESTA }\end{array}$} & \multirow{3}{*}{1079} & \multirow{3}{*}{---} \\
\hline & CNN & $9.72 \%$ (de error) & & & \\
\hline & NFL & $15.14 \%$ (de error) & & & \\
\hline \multirow{8}{*}{ Wen (2012) } & EIGENFACE & $93.87 \%$ & \multirow{8}{*}{ ORL } & \multirow{8}{*}{400} & \multirow{8}{*}{$112 \times 96$} \\
\hline & LDA & $93.80 \%$ & & & \\
\hline & PCA-SVM & $95.56 \%$ & & & \\
\hline & DCV-NN & $96.67 \%$ & & & \\
\hline & KDCV-NN & $97.75 \%$ & & & \\
\hline & DCV-RBF & $97.77 \%$ & & & \\
\hline & KDCV-RBF & $97.65 \%$ & & & \\
\hline & IDCV-SVM & $98.92 \%$ & & & \\
\hline $\begin{array}{l}\text { Kong y Zhang } \\
\text { (2011) }\end{array}$ & FLS-SVM (ICA) & $97.50 \%$ & $\mathrm{ORL}$ & 400 & $28 \times 28$ \\
\hline Devi et al., (2015) & R-KDA - SVM & $30 \%$ & UMIST & 564 & $220 \times 220$ \\
\hline $\begin{array}{l}\text { Olivares et al., } \\
\text { (2016) }\end{array}$ & LFDE - SVM & $97.92 \%$ & $A R$ & 9360 & --- \\
\hline Luo et al., (2013) & $P C A+L B P+S V M$ & $96.25 \%$ & PERSONAL & 50 & $24 \times 24$ \\
\hline Lin et al., (2016) & SVM & $97 \%$ & $\mathrm{ORL}$ & 509 & --- \\
\hline \multirow{3}{*}{ Kim et al., (2010) } & \multirow{3}{*}{ SVM-DA } & 8.5 \% (error mínimo) & FERET & 1702 & $56 \times 46$ \\
\hline & & 8.6 \% (error mínimo) & AR & 1680 & $56 \times 46$ \\
\hline & & 9.5 \% (error mínimo) & CMU-PIE & 1840 & $56 \times 46$ \\
\hline $\begin{array}{l}\text { Gumus et al. } \\
\text { (2010) }\end{array}$ & Wavelet-SVM & $89.38 \%$ & $\mathrm{ORL}$ & 400 & --- \\
\hline \multirow{2}{*}{ Li et al., (2011) } & \multirow{2}{*}{ MOUD-SVM } & \multirow{2}{*}{$96.67 \%$} & YALE & 165 & \multirow{2}{*}{---} \\
\hline & & & CAS-PEAL-R1 & 100 & \\
\hline $\begin{array}{l}\text { Zhou et al., } \\
\text { (2010) }\end{array}$ & $\mathrm{KSCH}$ & $100 \%$ & $\begin{array}{l}\text { MIT-CBCL } \\
\text { UMIST }\end{array}$ & 180 & --- \\
\hline \multirow{4}{*}{$\begin{array}{l}\text { Khan et al., } \\
(2012)\end{array}$} & \multirow{4}{*}{ SVM-NDA } & \multirow{4}{*}{$>98 \%$} & $\mathrm{ORL}$ & 400 & \multirow{4}{*}{---} \\
\hline & & & YALE & 165 & \\
\hline & & & ESSEX & 153 & \\
\hline & & & JAFFE & 213 & \\
\hline $\begin{array}{l}\text { Kasar et al. } \\
(2016)\end{array}$ & $\mathrm{BNP}+\mathrm{RBF}$ & $98.88 \%$ & ----- & $-\cdots--$ & ----- \\
\hline
\end{tabular}




\section{Results}

Based on the revised works, many techniques enable us to meet the objective of face recognition, among those that include: Supporting vectors, Core Component Analysis, Neighbouring $\mathrm{K}$-nn, Independent Component Analysis, Eigenface, many have also chosen to work with combinations such as PCA-SVM, DCV-NN, KDCV-NN, DCV-RBF, KDCV-RBF, IDCV-SVM, ICA-FLS_SVM, RKDA-SVM, GABOR-SVM, among others.

Efficiency has been achieved, especially when the SVM pattern classifier intervenes. This classifier works with kernel functions like Linear, Gaussian, polynomial, among others. It seems that the Gaussiano and Polynomial kernel have produced the best results. Of course, a very important element in this process is the databases used for it. In fact, the efficiency of recognition depends on many elements such as the training, the model obtained, the number of tests performed (ideally, as many tests as possible), the size of databases for training and testing, image resolution, and so on. In this sense, two works achieve the highest recognition rates, such as the Improved Discriminative Common Vectors (IDCV) technique used by SVM, achieve a recognition efficiency of $98.92 \%$ with an ORL, ATT and AR database, applying the Gaussian kernel, as well as the combined KSVM+KNDA work reaches $98.7 \%$ using a real processed database in laboratory and Kernels KSVM and KNDA.

\section{Conclusions and recommendations}

This bibliographic review work related to the recognition of computer imagery was carried out in-depth analysis of some related work, highlighting the databases used for their respective training and testing, techniques for extracting face characteristics such as PCA, KNN, RFB, among others, as well as pattern classifiers for recognition, combinations of techniques and classifiers such as ICA_SVM, KSCH+1 SVM, KSVM+KNDA, together with some kernels employed, such as Linear, Gaussiano, Polynomial, etc. It should be noted that many works were reviewed. Those that achieved the best results in recognition, of which it was seen that a SVM pattern classifier is always present, suggested that more attention needs to be paid to this classifier; according to the bibliographic revision this is a technique with a wide range of application in different fields such as: Physicians, Socio-economists, the prediction of natural phenomena, among others.

It has been observed that reconnaissance techniques work better at controlled times because the image obtained has better quality in resolution, but in practice it would be better to work with uncontrolled moments with high resolution cameras and be driven by highly efficient algorithms to obtain a good result in facial recognition. Thus, in the future, there is a new research of facial recognition aimed at designing algorithms for 3D facial recognition applied to uncontrolled moments.

Finally, the quality of face databases, filtering and feature extraction techniques, pattern classifier are important factors for a successful facial recognition process.

\section{Referencias}

Belén, A., Díaz, M., y Físicas, C. C. (2004). Reconocimiento Facial Automático mediante Técnicas de Visión Tridimensional Tesis Doctoral Reconocimiento Facial Automático mediante Técnicas de Visión Tridimensional Tesis Doctoral. 
Benavides, D. J., Jurado, F., y González, L. G. (2018). Data analysis and tools applied to modeling and simulation of a PV system in Ecuador (Análisis de datos y herramientas aplicadas al modelado y simulación de un sistema fotovoltaico en Ecuador), 1-12.

Benavente, R., Martínez, A. (2014). The AR Face Database. Computer Vision Center, (January 1998): 1-8.

Benitez-Garcia, G., Olivares-Mercado, J., Aguilar-Torres, G., Sanchez-Perez, G., y Perez-Meana, H. (2012). Face Identification Based on Contrast Limited Adaptive Histogram Equalization (CLAHE), (mayo 2015).

Cabello Pardos, E. (2003). Técnicas de reconocimiento facial mediante redes neuronales, 135. Retrieved from http://dialnet.unirioja.es/servlet/tesis?codigo=2586\&info=resumen\&idioma=SPA

Calle-López, D., Cornejo-Reyes, J., Pesántez-Avilés, F., y Rodas, M. (2018). Un sistema experto basado en minería de datos y programación entera lineal para soporte en la asignación de materias y diseño de horarios en educación superior (An expert system based on data mining and linear integer programming to support the timetabling design and courses assignment in higher education), 102-117.

Chang, C., \& Lin, C. (2013). LIBSVM : A Library for Support Vector Machines. ACM Transactions on Intelligent Systems and Technology (TIST), 2: 1-39. https://doi.org/10.1145/1961189.1961199

Correa, A. C., Salazar, A. E. S., y Ortiz, F. A. P. (2013). Reconocimiento de rostros y gestos faciales mediante un analisis de relecancia con imagenes 3D. Revista De Investigación, Desarrollo E Innovación, 4 (1): 7-20. https://doi.org/10.19053/20278306.2563

Deng, C. (2008). Four face databases in matlab format. Retrieved from http://www.cad.zju.edu.cn/home/ dengcai/Data/FaceData.html

Devi, H. S., Laishram, R., y Thounaojam, D. M. (2015). Face Recognition using R-KDA with Non-Linear SVM for Multi-View Database. Procedia Computer Science, 54, 532-541. https://doi.org/10.1016/j. procs.2015.06.061

Gao, W., Cao, B., Shan, S., Chen, X., Zhou, D., Zhan, X. (2008). The CAS-PEAL large-scale Chinese face database and baseline evaluations. IEE, 38 (1): 149-161.

Georghiades, A. (1997). Yale Face Database. Center for Computational Vision and Control, Yale.

González, M. (2007). Reconocimiento facial combinando técnicas 2D y 3D. Escola d'Enginyeria de Telecomunicació i Aeroespacial de Castelldefels. Barcelona.

Graham, D. B., y Allinson, N. (1998). Characterizing Virtual Eigensignatures for General Purpose Face Recognition. Face recognition: from theory to applications; $163 ; 446-456$. Springer.

Gumus, E., Kilic, N., Sertbas, A., y Ucan, O. N. (2010). Evaluation of face recognition techniques using PCA, wavelets and SVM. Expert Systems with Applications, 37 (9): 6404-6408. https://doi. org/10.1016/j.eswa.2010.02.079

Guo, G., Li, S. Z., y Chan, K. L. (2001). Support vector machines for face recognition. Image and Vision Computing, 19, 631-638. https://doi.org/10.1016/S0262-8856(01)00046-4

Jos, C., Alamo, D., Arnaldo, L., Calla, R., y P, L. J. F. (2015). Efficient approach for interest points detection in non-rigid shapes.

Kasar, M. M., Bhattacharyya, D., y Kim, T. (2016). Face Recognition Using Neural Network : A Review, 10 (3): 81-100.

Khan, N. M., Ksantini, R., Ahmad, I. S., \& Boufama, B. (2012). A novel SVM+NDA model for classification with an application to face recognition. Pattern Recognition, 45 (1): 66-79. https://doi.org/10.1016/j. patcog.2011.05.004

Kim, S.-K., Park, Y. J., Toh, K.-A., y Lee, S. (2010). SVM-based feature extraction for face recognition. Pattern Recognition, 43 (8): 2871-2881. https://doi.org/10.1016/j.patcog.2010.03.008

Kong, R., y Zhang, B. (2011). A New Face Recognition Method Based on Fast Least Squares Support Vector Machine. Physics Procedia, 22: 616-621. https://doi.org/10.1016/j.phpro.2011.11.095

Li, W., Liu, L., y Gong, W. (2011). Multi-objective uniform design as a SVM model selection tool for face recognition. Expert Systems with Applications, 38 (6): 6689-6695. https://doi.org/10.1016/j. eswa.2010.11.066 
Lin, W.-H., Wang, P., y Tsai, C.-F. (2016). Face recognition using support vector model classifier for user authentication. Electronic Commerce Research and Applications, 18 (February), 1-12. https:// doi.org/10.1016/j.elerap.2016.01.005

Luo, Y., Wu, C. M., y Zhang, Y. (2013). Facial expression recognition based on fusion feature of PCA and LBP with SVM. Optik, 124 (17): 2767-2770. https://doi.org/10.1016/j.ijleo.2012.08.040

Lyons, M. J. (1999). Automatic classification of single facial images. IEEE Transactions on Pattern Analysis and Machine Intelligence, 21 (12): 1357-1362. https://doi.org/10.1109/34.817413

Michel, A., Aleaga, L., Fernando, E., Garcés, M., y García, A. C. (2015). Sistema automatizado para la predicción de flujo de carga en subestaciones eléctricas mediante redes neuronales artificiales (Automated system for load flow prediction in power substations using artificial neural networks), 20-35.Moreno, A.B., y Sanchez, A. (2004). GavabDB: A 3D Face Database. Proceedings 2nd COST Workshop on Biometrics on the Internet: Fundamentals, Advances and Applications. Vigo. pp. 77-82.

Moreno, A. B., Sanchez, A., Vélez, J. y Díaz, J. (2005). Face recognition using 3D local geometrical features: PCA vs. SVM. Image and Signal ..., (1): 185-190. https://doi.org/10.1109/ispa.2005.195407

Olivares-Mercado, J., Toscano-Medina, K., Sánchez-Pérez, G., Nakano-Miyatake, M., y Pérez-Meana, H. (2016). Modifications to the Eigenphases Method for Face Recognition Based on SVM. Ingeniería, Investigación y Tecnología, 17 (1): 119-129. https://doi.org/10.1016/j.riit.2016.01.011

Peng, X. (2010). An efficient twin support vector machine for regression, PublMed, 23 (3): 365-372.

Phillips, P., Moon, H., Rizvi, S. y Rauss, P. (2000). The FERET evaluation methodology for face recognition algorithms, 22 (10): 1090-1104.

Pradhan, B. (2013). A comparative study on the predictive ability of the decision tree, support vector machine and neuro-fuzzy models in landslide susceptibility mapping using GIS, 51: 350-365.

Sim, T., Baker, S., y Bsat, M. (2003). The \{CMU\} pose, illuminlation, and expression database. Pami, 25 (12): 1615-1618. https://doi.org/10.1109/AFGR.2002.1004130

Spacek, L. (2008). Face recognition data ESSEX.

Stewart Bartlett, M. (1999). Face image analysis by unsupervised learning. Boston: Springer.

Valdes, M. M. L., Aleaga, A. M. L., y Vidal, G. G. (2014). Redes neuronales artificiales en la predicción de insolvencia. Un cambio de paradigma ante recetas tradicionales de prácticas empresariales (Artificial Neural Networks in the prediction of insolvency. A paradigm shift to traditional business practices recipes, 14: 38-58.

Vapnik, V. N. (1998). Statistical Learning Theory. Adaptive and Learning Systems for Signal Processing. Communications and Control, 2: 1-740. https://doi.org/10.2307/1271368

Wen, Y. (2012). An improved discriminative common vectors and support vector machine based face recognition approach. Expert Systems with Applications, 39 (4): 4628-4632. https://doi. org/10.1016/j.eswa.2011.09.119

Weyrauch, B., Heisele, B., y Blanz, V. (2004). Component-based Face Recognition with 3D Morphable Models. IEE: 0-4.

Yu, H., y Yang, J. (2001). A direct LDA algorithm for high-dimensional data-with application to face recognition. Pattern Recognition, 34 (February): 2067-2070. https://doi.org/10.1016/S00313203(00)00162-X

Z. Qi, Y. Tian, Y. S. (2013). Robust twin support vector machine for pattern classification, 305-316.

Zhou, X., Jiang, W., Tian, Y., Y Shi, Y. (2010). Kernel subclass convex hull sample selection method for SVM on face recognition. Neurocomputing, 73 (10-12): 2234-2246. https://doi.org/10.1016/j.neucom.2010.01.008 